Louisiana State University

LSU Digital Commons

Faculty Publications

Department of Chemistry

$11-21-2019$

\title{
Angle-dependent strong-field ionization of halomethanes
}

Péter Sándor

University of Virginia

Adonay Sissay

Louisiana State University

François Mauger

Louisiana State University

Mark W. Gordon

University of Virginia

T. T. Gorman

The Ohio State University

See next page for additional authors

Follow this and additional works at: https://digitalcommons.Isu.edu/chemistry_pubs

\section{Recommended Citation}

Sándor, P., Sissay, A., Mauger, F., Gordon, M., Gorman, T., Scarborough, T., Gaarde, M., Lopata, K., Schafer, K., \& Jones, R. (2019). Angle-dependent strong-field ionization of halomethanes. Journal of Chemical Physics, 151 (19) https://doi.org/10.1063/1.5121711

This Article is brought to you for free and open access by the Department of Chemistry at LSU Digital Commons. It has been accepted for inclusion in Faculty Publications by an authorized administrator of LSU Digital Commons.

For more information, please contact ir@lsu.edu. 


\section{Authors}

Péter Sándor, Adonay Sissay, François Mauger, Mark W. Gordon, T. T. Gorman, T. D. Scarborough, Mette B. Gaarde, Kenneth Lopata, K. J. Schafer, and R. R. Jones 


\section{Angle-dependent strong-field ionization of halomethanes}

Cite as: J. Chem. Phys. 151, 194308 (2019); https://doi.org/10.1063/1.5121711

Submitted: 26 July 2019 • Accepted: 05 November 2019 • Published Online: 21 November 2019

Péter Sándor, Adonay Sissay, (D) François Mauger, et al.
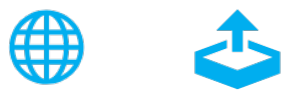

\section{ARTICLES YOU MAY BE INTERESTED IN}

Relativistic four-component linear damped response TDDFT for electronic absorption and circular dichroism calculations

The Journal of Chemical Physics 151, 194112 (2019); https://doi.org/10.1063/1.5128564

Analytical gradients and derivative couplings for dynamically weighted complete active space self-consistent field

The Journal of Chemical Physics 151, 201101 (2019); https://doi.org/10.1063/1.5130997

Saturated Raman scattering for sub-diffraction-limited imaging

The Journal of Chemical Physics 151, 194201 (2019); https://doi.org/10.1063/1.5128874

Challenge us.

What are your needs for periodic signal detection?

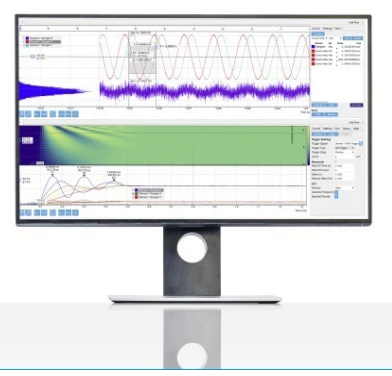

Zurich - Instruments 


\title{
Angle-dependent strong-field ionization of halomethanes
}

\author{
Cite as: J. Chem. Phys. 151, 194308 (2019); doi: 10.1063/1.5121711 \\ Submitted: 26 July 2019 - Accepted: 5 November 2019 • \\ Published Online: 21 November 2019
}

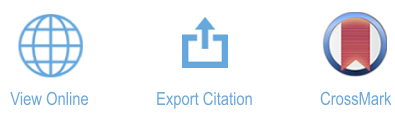

\author{
Péter Sándor, ${ }^{1}$ (D) Adonay Sissay, ${ }^{2}$ François Mauger, ${ }^{3}$ (D) Mark W. Gordon, ${ }^{1}$ T. T. Gorman, ${ }^{4}$ T. D. Scarborough, \\ Mette B. Gaarde, ${ }^{3}$ Kenneth Lopata, ${ }^{2, a)}$ (D) K. J. Schafer, ${ }^{3}$ and R. R. Jones ${ }^{1, b)}$
}

\author{
AFFILIATIONS \\ 'Department of Physics, University of Virginia, Charlottesville, Virginia 22904, USA \\ ${ }^{2}$ Department of Chemistry, Louisiana State University, Baton Rouge, Louisiana 70803, USA \\ ${ }^{3}$ Department of Physics and Astronomy, Louisiana State University, Baton Rouge, Louisiana 70803, USA \\ ${ }^{4}$ Department of Physics, The Ohio State University, Columbus, Ohio 43210, USA
}

\begin{abstract}
a) Also at: Center for Computation and Technology, Louisiana State University, Baton Rouge, Louisiana 70803, USA.
${ }^{b}$ Electronic mail: rrj3c@virginia.edu
\end{abstract}

\begin{abstract}
We study, experimentally and theoretically, the ionization probability of singly halogenated methane molecules, $\mathrm{CH}_{3} \mathrm{Cl}_{\text {and }} \mathrm{CH}_{3} \mathrm{Br}$, in intense linearly polarized $800 \mathrm{~nm}$ laser pulses as a function of the angle between the molecular axis and the laser polarization. Experimentally, the molecules are exposed to two laser pulses with a relative time delay. The first, weaker pulse induces a nuclear rotational wave packet within the molecules, which are then ionized by the second, stronger pulse. The angle-dependent ionization yields are extracted from fits of the measured delay-dependent ionization signal to a superposition of moments of the rotational wave packet's angular distribution. Angle-dependent strong-field ionization (SFI) yields are also calculated using time-dependent density functional theory. Good agreement between measurements and theory is obtained. Interestingly, we find a marked difference between the angle-dependence of the ionization yields for these two halomethane species despite the similar structure of their highest occupied molecular orbitals. Calculations reveal that these differences are a result of multichannel $\left(\mathrm{CH}_{3} \mathrm{Cl}\right)$ vs single-channel $\left(\mathrm{CH}_{3} \mathrm{Br}\right)$ ionization and of increased hole localization on $\mathrm{Br}$ vs $\mathrm{Cl}$. By adding calculations for $\mathrm{CH}_{3} \mathrm{~F}$, we can discern clear trends in the ionization dynamics with increasing halogen mass. These results are illustrative, as chemical functionalization and molecular alignment are likely to be important parameters for initiating and controlling charge migration dynamics via SFI.
\end{abstract}

Published under license by AIP Publishing. https://doi.org/10.1063/1.5121711

\section{INTRODUCTION}

For molecules exposed to intense low-frequency radiation, the strong-field ionization (SFI) rates can depend nontrivially on the alignment/orientation of the molecule relative to the direction of the applied field at the instant of ionization. ${ }^{1-12}$ Because ionization triggers many strong-field processes of current interest, from high-harmonic generation and attosecond pulse generation to laser-induced electron diffraction for time-resolved molecular imaging, ${ }^{13-19}$ it is important to understand the ionization anisotropy in molecular systems of interest. More interestingly, however, the alignment/orientation of the molecule relative to the laser polarization influences the degree to which various molecular channels contribute to the ionization process and the subsequent coherent hole dynamics in the molecular ion. As such, the ability to control molecular alignment/orientation in the laboratory and to accurately simulate the collective electronic response to the intense laser are critical capabilities for exploring ultrafast electronic processes in molecules. Here, we extend our investigation of angle-dependent SFI from linear ${ }^{20}$ to symmetric top molecules. We describe measurements of intensity- and angle-dependent SFI of halomethanes using $800 \mathrm{~nm}$ laser light and compare those results to calculations that not only predict the ionization yields but also provide deeper insight into the mechanisms underlying SFI in these systems. The theoretical picture of SFI we present, based upon time-dependent density functional theory (TDDFT), is brought into clearer focus 
by adding calculations for the lightest halomethane, $\mathrm{CH}_{3} \mathrm{~F}$. With this, we can discern clear trends in the ionization dynamics with increasing halogen mass.

Ionization in strong AC fields is potentially a very complex process, including density polarization due to the strong fields, as well as hole dynamics within the molecule. Time-dependent simulations such as those we use naturally include these effects, though it is a significant challenge to extract their precise contribution to the dynamics. In this paper, we build on our previous methodology ${ }^{20}$ for interpreting the ionization in terms of subcycle ionization yields and time-dependent hole localization. This enables a direct comparison to the experimental yields using the same laser parameters, as well as interpretation in terms of what regions of the molecule contribute to ionization and where the hole localizes following ionization. We note that the effect of halogen substitution on strong-field ionization in the $\mathrm{CH}_{3} \mathrm{X}$ family has been previously studied theoretically using time-dependent configuration-interaction. ${ }^{21}$ This study, which used strong, adiabatically switched-on static fields, demonstrated that $\mathrm{CH}_{3} \mathrm{~F}$ ionizes predominantly from the methyl group, $\mathrm{CH}_{3} \mathrm{I}$ from the iodine, and $\mathrm{CH}_{3} \mathrm{Cl}$ and $\mathrm{CH}_{3} \mathrm{Br}$ from both groups of the molecule. Though these results are most comparable to ionization in the long wavelength limit, we find that the progression from methyl to halogen-dominated angle-dependent ionization is consistent with our calculations at $800 \mathrm{~nm}$.

In the following sections, we first briefly review our experimental and theoretical approach and present our new results for chloromethane and bromomethane. We show that despite the substantial similarities in the electronic structure of these two species, there are substantial differences in the angle-dependent ionization yields. The calculations guide our identification of the causes of those differences and give insight into the postionization hole dynamics that are not directly accessible to the experiment. We conclude with a discussion of the use of angle-dependent SFI to induce and perhaps probe charge localization dynamics in molecules.

\section{EXPERIMENTAL APPROACH}

Details of the experimental setup are described in Ref. 20. Briefly, two nearly collinear, linearly polarized, $800 \mathrm{~nm}$ laser pulses from a $1 \mathrm{kHz}$ repetition rate multipass Ti:Sapphire amplifier are focused on a rotationally cold molecular beam $\left(500 \mathrm{ppm} \mathrm{CH}_{3} \mathrm{Cl}\right.$ or $\mathrm{CH}_{3} \mathrm{Br}$ in $\mathrm{He}$ buffer expanded through an Even-Lavie valve synchronously pulsed at $1 \mathrm{kHz}$ ). The laser pulses have a variable timedelay, $\tau$, between them; the first (75 fs duration, $15-20 \mathrm{TW} / \mathrm{cm}^{2}$ ) induces transient molecular alignment but no ionization, while the second ( 37 fs duration) ionizes the target. Ions produced by the laser-molecule interaction are collected using a static electric field and detected with a microchannel plate stack. The time-dependent voltage from the detector is amplified and digitized, and different ion species are identified based on their time-of-flight. To eliminate signal variations associated with (small) long-term drifts in sample density and laser intensity, we measure the normalized ion yield vs $\tau$ for each species of interest. Specifically, the signal in the associated time-of-flight peak is integrated, and the ratio of the integrated signals with the alignment pulse on and off is recorded. The normalized ion yields from 100 to 200 delay scans are combined to obtain the delay-dependent, normalized ion yield, $Y(\tau)$, for a given ionization laser intensity.
The peak ionization laser intensity is calibrated as described in Ref. 20. This intensity calibration is used, without any additional rescaling, to directly compare the measured and calculated angle-dependent ionization yields from $\mathrm{CH}_{3} \mathrm{Cl}$ and $\mathrm{CH}_{3} \mathrm{Br}$.

\section{EXPERIMENTAL RESULTS}

Figure 1 shows the normalized ion yield measurements, $Y(\tau)$, for $\mathrm{CH}_{3} \mathrm{Cl}^{+}$and $\mathrm{CH}_{3} \mathrm{Br}^{+}$at two different intensities. The alignment pulse parameters were held approximately constant for all data sets. Clear variations in the normalized ionization yields are observed at delays near the quarter-, half-, and full-rotational revival times, 9.5 ps, 19 ps, and 38 ps, respectively, for $\mathrm{CH}_{3} \mathrm{Cl}^{+}$. For $\mathrm{CH}_{3} \mathrm{Br}^{+}$, the quarter revival is not as pronounced, but the half and full revivals are clearly identified around 26 ps and 52 ps, respectively. The revival structures for the two species exhibit striking differences. For chloromethane, the polarities of the two peaks within the full revival are the same, as are the two peaks at the half revival [see Figs. 1(a) and 1(b)]. For bromomethane, the two peaks within the full revival (and also at the half revival) have the opposite polarity [Figs. 1(c) and 1(d)]. The rotational wave packets created in the two molecules are very similar, so the observed dissimilarities in the time-dependent yields reflect substantial qualitative differences in the angle-dependent ionization rates for the two molecules. We return to this point in Sec. IV A.

Figure 2 shows the unaligned $\mathrm{CH}_{3}{ }^{35} \mathrm{Cl}^{+}$and $\mathrm{CH}_{3}{ }^{79} \mathrm{Br}^{+}$yields as a function of laser intensity. At the lowest intensities, the yields grow rapidly with increasing laser intensity, as expected for tunneling or high-order multiphoton ionization. At the highest intensity, the ion yields are fully saturated, comparable in magnitude, and are essentially independent of laser intensity. Following Hankin et al., ${ }^{22}$ we separately fit the high- and low-intensity portions of the measured yields to curves of the form $\alpha I^{\beta}$, where $I$ is the peak intensity of the ionization pulse. We define the saturation intensity as the point of intersection of those two curves. The data in Fig. 1 were collected below and near the respective saturation intensities, $I_{\mathrm{CH}_{3} \mathrm{Cl}} \simeq 82 \mathrm{TW} / \mathrm{cm}^{2}$ and $I_{\mathrm{CH}_{3} \mathrm{Br}} \simeq 62 \mathrm{TW} / \mathrm{cm}^{2}$. The measured $Y(\tau)$ curves in Fig. 1 suggest that the angle-dependent SFI rates for $\mathrm{CH}_{3} \mathrm{Br}\left(\mathrm{CH}_{3} \mathrm{Cl}\right)$ are nearly independent of (weakly dependent on) laser intensity below saturation. Somewhat surprisingly, the delay dependent structures in $\mathrm{CH}_{3} \mathrm{Cl}^{+}$curves are actually more pronounced near the saturation intensity [compare Figs. 1(a) and $1(\mathrm{~b})]$.

Unlike some previous studies of angle-dependent ionization of $\mathrm{CH}_{3} \mathrm{I}$, we restrict our measurements to nondissociative single ionization channels at intensities below saturation. ${ }^{23,24}$ Even so, some dissociative ionization does occur. Under the conditions used to collect the data in Fig. 1, the ratios of ion fragments to nondissociated molecular ion yields are as follows: (near $45 \mathrm{TW} / \mathrm{cm}^{2}$ ) $\sum_{n=0}^{3} \mathrm{CH}_{n}^{+}: \mathrm{CH}_{3} \mathrm{Cl}^{+} \simeq 11 \%, \mathrm{Cl}^{+}: \mathrm{CH}_{3} \mathrm{Cl}^{+}<1 \%, \sum_{n=0}^{3} \mathrm{CH}_{n}^{+}: \mathrm{CH}_{3} \mathrm{Br}^{+}$ $\simeq 6 \%, \mathrm{Br}^{+}: \mathrm{CH}_{3} \mathrm{Br}^{+}<1 \% ;\left(\right.$ near $\left.60 \mathrm{TW} / \mathrm{cm}^{2}\right) \sum_{n=0}^{3} \mathrm{CH}_{n}^{+}: \mathrm{CH}_{3} \mathrm{Cl}^{+}$ $\simeq 19 \%, \mathrm{Cl}^{+}: \mathrm{CH}_{3} \mathrm{Cl}^{+}<1 \%, \sum_{n=0}^{3} \mathrm{CH}_{n}^{+}: \mathrm{CH}_{3} \mathrm{Br}^{+} \simeq 16 \%, \mathrm{Br}^{+}: \mathrm{CH}_{3} \mathrm{Br}^{+}$ $\simeq 3 \%$. At higher intensities, dissociative and multiple ionization become even more significant. When produced with a high probability, these competing channels can preferentially deplete the parent ion yield at some angles, distorting the measured angular dependence. It is worth noting, however, that dissociative and multiple 


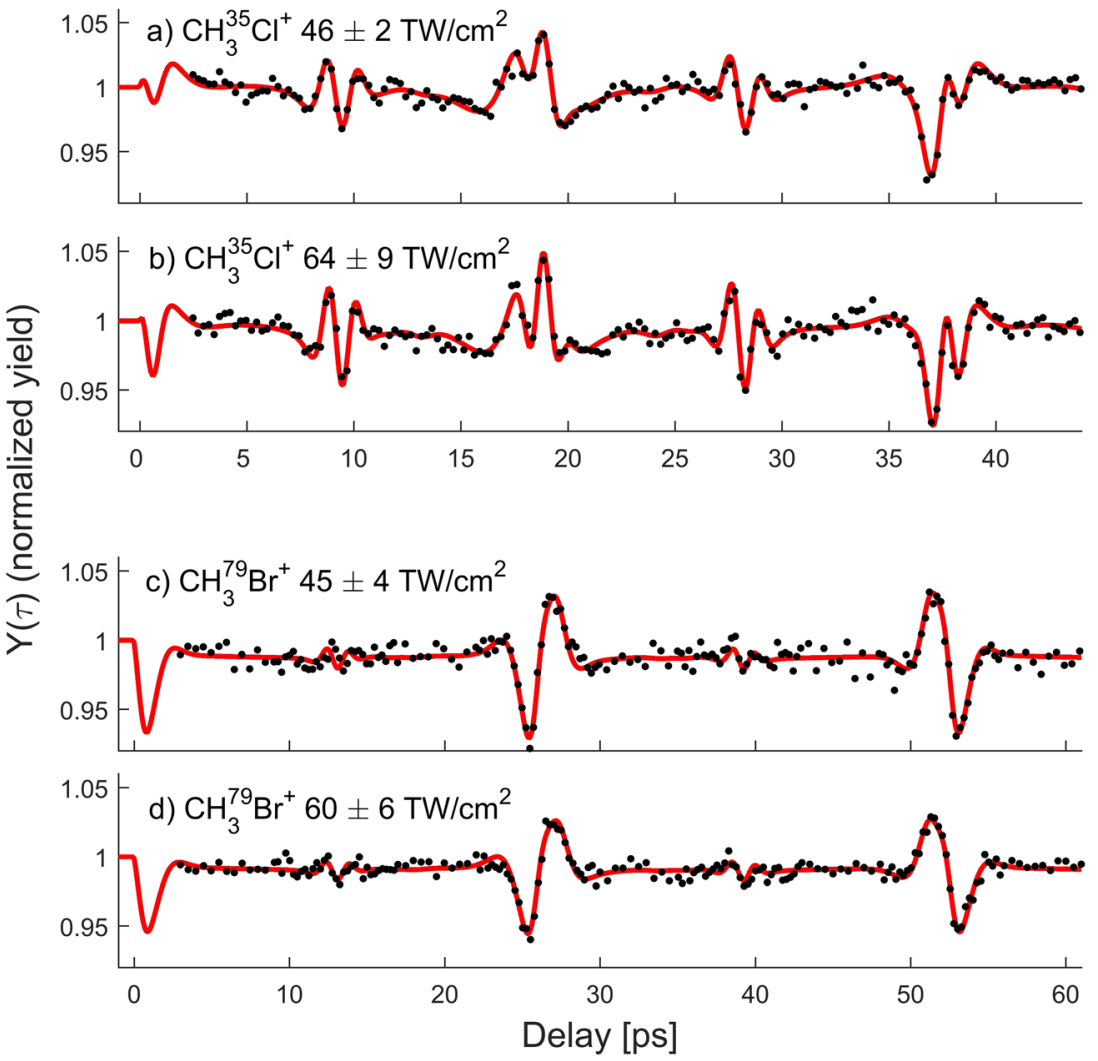

ionization channels have little impact on the saturation intensity determination in both species. Inclusion of the fragment ion and (small) parent dication yields with those of singly ionized parent molecules results in saturation intensities that differ by $5 \%$ or less relative to those extracted using the parent ion yields alone.

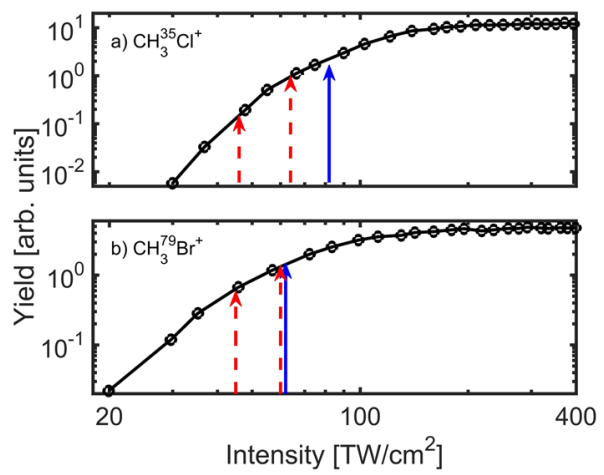

FIG. 2. $\mathrm{CH}_{3}{ }^{35} \mathrm{Cl}^{+}$(a) and $\mathrm{CH}_{3}{ }^{79} \mathrm{Br}^{+}$(b) yields vs ionizing laser intensity for unaligned molecules. Vertical red dashed arrows show the intensities at which the data in Fig. 1 were collected. Vertical blue arrows show the experimentally determined saturation intensities. The intensity axis is calibrated as described in Ref. 20.

\section{ANALYSIS AND DISCUSSION}

The angle-dependent molecular ionization yield $\Upsilon(\theta)$ is extracted from the delay dependent yield $Y(\tau)$ (Fig. 1) by fitting those data to a linear combination of delay-dependent moments of the rotational probability distribution, $M_{k}(\tau)$, calculated $a$ priori. Here, $\theta$ is the angle between the alignment laser polarization and the $\mathrm{C}-\mathrm{X}(\mathrm{X}=$ halogen $)$ axis in the molecule. For a detailed description of the procedure, see Ref. 20. To summarize, the delay-dependent yield is expressed as

$$
\begin{aligned}
Y(\tau) & =\int_{0}^{\pi} \Upsilon(\theta) S(\theta, \tau) \sin (\theta) d \theta \\
& \equiv \sum_{k} a_{k} M_{k}(\tau),
\end{aligned}
$$

where $S(\theta, \tau)$ is the delay-dependent, ensemble average of the angular probability distribution. It is calculated with high accuracy by numerical integration of the time-dependent Schrödinger equation for the rotational wave packet. ${ }^{3}$ The solution assumes an initial thermal ensemble of (nearly) rigid molecular rotors with rotational temperature $T_{r o t}$ and an alignment laser pulse with a Gaussian envelope, duration $\tau_{0}$, and peak intensity $I_{0} \cdot{ }^{3,12,25}$ It requires, among others, several molecule-specific constants as inputs, such as the rotational constants (A and $\mathrm{B}$ ) and polarizability anisotropies $(\Delta \alpha)$, which are listed in Table I. Based on FROG measurements, ${ }^{26}$ our alignment laser pulse is known to have a near Gaussian temporal profile 
TABLE I. Relevant molecular properties: polarizability anisotropy $(\Delta \alpha)$ and rotational constants $A$ and $B$.

\begin{tabular}{lcll}
\hline \hline Molecule & $\Delta \alpha$ [a.u.] & $\mathrm{A}\left(\mathrm{cm}^{-1}\right)$ & $\mathrm{B}\left(\mathrm{cm}^{-1}\right)$ \\
\hline $\mathrm{CH}_{3}^{35} \mathrm{Cl}$ & $11.5^{29}$ & $5.097^{30}$ & $0.4434^{30}$ \\
$\mathrm{CH}_{3}{ }^{79} \mathrm{Br}$ & $13.18^{29}$ & $5.2463^{30}$ & $0.31916^{31}$ \\
\hline \hline
\end{tabular}

with a full-width at half maximum (FWHM) of 75 fs. Rather than rely on estimates of $I_{0}$ and $T_{r o t}$, we allow these parameters to vary along with the expansion coefficients $a_{k}$ to obtain the best overall fit to $Y(\tau)$. The recovered values for $I_{0}\left(15-20 \mathrm{TW} / \mathrm{cm}^{2}\right)$ and $T_{\text {rot }}$ (5-8 K for $\mathrm{CH}_{3} \mathrm{Cl}$ and $9-11 \mathrm{~K}$ for $\mathrm{CH}_{3} \mathrm{Br}$ ) are in reasonable agreement with those estimated from measured laser beam properties and from previous performance characteristics of the Even-Lavie valve, ${ }^{20,27,28}$ respectively.

The delay-dependent moments $\mathrm{M}_{k}(\tau)$ are calculated as

$$
M_{k}(\tau)=\int_{0}^{\pi} f_{k}(\theta) S(\theta, \tau) \sin (\theta) d \theta
$$

where $f_{k}(\theta)$ are a set of real functions on which $\Upsilon(\theta)$ is expanded with coefficients $a_{k}$. Rather than attempt to construct $Y(\tau)$ from a complete, orthonormal basis, we choose a small set of $f(k)$ functions that individually satisfy the two criteria noted below and enable satisfactory fitting within the measurement signal-to-noise. The first constraint is the symmetry imposed by the alignment: $\Upsilon(\theta)=\Upsilon(\pi$ $-\theta) \geq 0$ for $0 \leq \theta \leq \pi$. The second criterion is based on the observation that the $\mathrm{CH}_{3} \mathrm{Br}$ ionization yield is the lowest when the laser polarization is parallel to the molecular axis. Thus, as in Ref. 20, we choose $f_{0} \equiv 1$ and $f_{k}(\theta)=\sin ^{2}(k \theta)$ for $\mathrm{k} \geq 1$. Beyond providing a good description of the data with the inclusion of only a few terms, these functions allow for a structure with a specific $2 k$-fold symmetry. For example, the $\sin ^{2}(\theta)$ and $\sin ^{2}(2 \theta)$ terms allow for dipole and quadrupole characters, respectively. The same set of basis functions was used in the case of $\mathrm{CH}_{3} \mathrm{Cl}^{+}$despite the different angle-dependent structure. The ionization yield is then given by

$$
\begin{aligned}
\Upsilon(\theta) & =\sum_{k} a_{k} f_{k}(\theta) \\
& =a_{0}+a_{1} \sin ^{2}(\theta)+a_{2} \sin ^{2}(2 \theta)+a_{3} \sin ^{2}(3 \theta)+\cdots .
\end{aligned}
$$

The coefficients $a_{k}$ are found by performing a nonlinear leastsquares fit on the measured $Y(\tau)$. The statistical F-test is used to determine the number of statistically significant terms in the expansion of $\Upsilon(\theta)$. For both $\mathrm{CH}_{3} \mathrm{Cl}^{+}$and $\mathrm{CH}_{3} \mathrm{Br}^{+}$, only three terms are needed, up to and including $\sin ^{2}(2 \theta)$. Apart from the three coefficients $a_{0}, a_{1}$, and $a_{2}$, two additional parameters are allowed to vary during the fitting process. ${ }^{20}$ The first makes a slight $(\approx 0.5 \%)$ multiplicative correction to the measured delay between the alignment and ionization pulses, due to the uncertainty in the position encoder on the translation stage. The second rescales the measured yield by $<1 \%$ to take into account the fact that, even though the alignment pulse does not induce ionization on its own, it can result in some electronic excitation which enhances the yield produced by the ionization pulse. The experimental zero time delay is determined absolutely by the enhanced ionization signal obtained when the two pulses are temporally overlapped. The best fit coefficients are determined by searches in the $\left(T_{r o t}, I_{0}\right)$ space, ${ }^{20}$ and those corresponding to the curves in Fig. 1 are presented in Table II.

\section{A. Angle-dependent yields}

Plots of $\Upsilon(\theta)$, obtained through the approach outlined in Sec. IV, are shown in Fig. 3. We separately normalize the yields at each intensity such that $\int_{0}^{\pi} \Upsilon(\theta) \sin (\theta) d \theta=1$. For $\mathrm{CH}_{3} \mathrm{Cl}^{+}$ [Figs. 3(a) and 3(b)], the constant and quadrupole terms dominate the angular distribution, resulting in a distinctive "cloverleaf" shape. Interestingly, for $\mathrm{CH}_{3} \mathrm{Cl}^{+}$, the lobes at $45^{\circ}$ and $135^{\circ}$ are more pronounced for the higher ionization intensity. This seems counterintuitive, since closer to the saturation intensity, one might expect the contrast of the angular features to diminish. For $\mathrm{CH}_{3} \mathrm{Br}^{+}$[Figs. 3(c) and $3(\mathrm{~d})]$, the angular distributions are distinctly different from those for $\mathrm{CH}_{3} \mathrm{Cl}^{+}$. Here, the constant and dipole components dominate (see Table II) leading to "peanut"-shaped distributions that are oriented perpendicular to the molecular axis and are essentially intensity independent.

One might expect a strong correlation between the angledependent yields $\Upsilon(\theta)$ and the shape of the highest occupied molecular orbital (HOMO). Ohmura et al., 32 showed that there is a clear progression of the $\mathrm{HOMO}$ orbital shape for a series of singly halogenated methanes from $\mathrm{CH}_{3} \mathrm{~F}$ through $\mathrm{CH}_{3} \mathrm{Cl}$ and $\mathrm{CH}_{3} \mathrm{Br}$ to $\mathrm{CH}_{3} \mathrm{I}$. While all four HOMOs have essentially a quadrupole character, the relative size of the molecular orbital lobes on the methyl end monotonically decreases relative to that of the halogen end, with the lobes having a roughly equal size for $\mathrm{CH}_{3} \mathrm{Cl}$. One might speculate that in our measurement, the quadrupole character of the angular distribution is the strongest for $\mathrm{CH}_{3} \mathrm{Cl}$ because the spatial distribution of the $\mathrm{HOMO}$ is more balanced.

For a more quantitative understanding of our experimental results, we have performed time-dependent density functional theory (TDDFT) calculations of SFI of $\mathrm{CH}_{3} \mathrm{Cl}$ and $\mathrm{CH}_{3} \mathrm{Br}$ under

TABLE II. Estimated coefficients $a_{k}$ (mean \pm standard deviation) in the best fit expansion of $\Upsilon(\theta)$ obtained from fits to $Y(\tau)$ at different ionization laser intensities.

\begin{tabular}{lccc}
\hline \hline Ionic species, intensity $\left(\mathrm{TW} / \mathrm{cm}^{2}\right)$ & $a_{0}$ & $a_{1}$ & $a_{2}$ \\
\hline $\mathrm{CH}_{3}^{35} \mathrm{Cl}^{+}, 46 \pm 2$ & $2.57 \pm 0.03$ & $0.06 \pm 0.03$ & $1.00 \pm 0.04$ \\
$\mathrm{CH}_{3}^{35} \mathrm{Cl}^{+}, 64 \pm 9$ & $2.05 \pm 0.04$ & $0.44 \pm 0.03$ & $1.49 \pm 0.05$ \\
$\mathrm{CH}_{3}^{79} \mathrm{Br}^{+}, 45 \pm 4$ & $2.18 \pm 0.07$ & $1.09 \pm 0.05$ & $0.44 \pm 0.08$ \\
$\mathrm{CH}_{3}^{79} \mathrm{Br}^{+}, 60 \pm 6$ & $2.20 \pm 0.06$ & $1.02 \pm 0.04$ & $0.50 \pm 0.07$ \\
\hline \hline
\end{tabular}



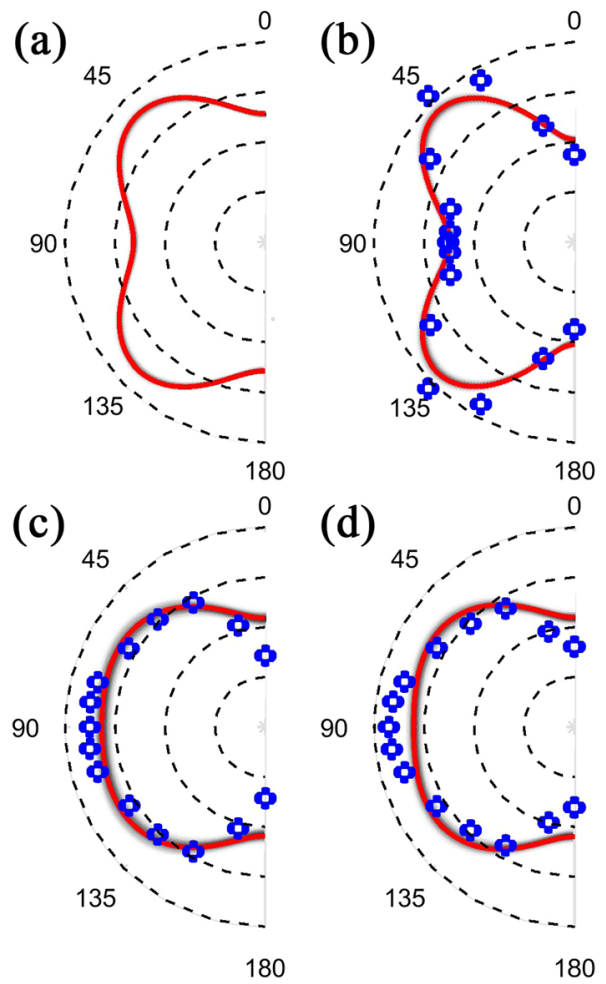

FIG. 3. Angular dependence of the ionization yield, $\Upsilon(\theta)$, plotted for $\mathrm{CH}_{3} \mathrm{Cl}^{+}$at ionization intensities of (a) 46 and (b) $64 \mathrm{TW} / \mathrm{cm}^{2}$ and for $\mathrm{CH}_{3} \mathrm{Br}^{+}$at ionization intensities of (c) 45 and (d) $60 \mathrm{TW} / \mathrm{cm}^{2}$. Red curves show the best fit to measurements of the normalized time-dependent yields $Y(\tau)$. The grayscale shadings, barely visible in (a) and (b), illustrate the experimental uncertainty distributions associated with the standard deviation of the coefficients $a_{k}$ obtained from the fitting. Blue symbols show the results of the TDDFT calculations described in the text.

the same conditions as our experiments. Additionally, we include simulations of fluoromethane $\left(\mathrm{CH}_{3} \mathrm{~F}\right)$, which is not studied experimentally here, to explore the systematics of the dependence of ionization on chemical functionalization. The results for the angledependent ionization yields, shown in Fig. 3, are in very good agreement with measurements. As described in Sec. VI, we take advantage of this agreement to extract possible mechanisms responsible for the different ionization anisotropies and to predict the degree of charge localization on the molecular cation immediately following ionization. The latter is a critical parameter for the initiation and observability of charge migration dynamics triggered by SFI.

\section{SIMULATION APPROACH}

The simulation approach we use is the same as that used previously. ${ }^{20}$ Briefly, we employ real-time TDDFT $^{33-38}$ implemented in NWChem ${ }^{39}$ with atom-centered Gaussian basis functions. ${ }^{40} \mathrm{~A}$ complex absorbing potential (CAP) emulates the ionization continuum by absorbing the outgoing electron flux. ${ }^{41-44}$ The CAP takes the form of a smoothly increasing imaginary potential centered on each atom starting at a distance $R_{0}$ (see Ref. 43 for details). For
TABLE III. TDDFT simulation parameters. Here, apVTZ = aug-cc-pvtz and \&\& denotes the large auxiliary Schlegel absorbing basis. ${ }^{41}$ For the tuned LC-PBE* functional, the global hybrid parameters $\alpha$ and $\beta$ are dimensionless, and the range-separation parameter $\mu$ is in a.u. ${ }^{-1}$.

\begin{tabular}{lccc}
\hline \hline Molecule & Basis set & LC-PBE0* & CAP $R_{0}(\AA)$ \\
\hline & H, F: apVTZ & $\begin{array}{l}\alpha=0.55 \\
\beta=0.45\end{array}$ & 7.0 \\
$\mathrm{CH}_{3} \mathrm{~F}$ & C: apVTZ\&\& & $\mu=0.20$ & \\
& & $\alpha=0.60$ & \\
& $\mathrm{H}, \mathrm{Cl}:$ apVTZ & $\beta=0.40$ & 7.0 \\
$\mathrm{CH}_{3} \mathrm{Cl}$ & $\mathrm{C}:$ apVTZ\&\& & $\mu=0.15$ & \\
& & $\alpha=0.70$ & \\
& $\mathrm{H}$, Br: apVTZ & $\beta=0.30$ & 7.0 \\
$\mathrm{CH}_{3} \mathrm{Br}$ & $\mathrm{C}:$ apVTZ\&\& & $\mu=0.30$ & \\
& &
\end{tabular}

the exchange-correlation functional, we employ the LC-PBE0* functional, which is a range-separated hybrid functional, ${ }^{45}$ optimally tuned $^{46,47}$ to enforce Koopman's theorem (i.e., the HOMO eigenvalue equals the ionization energy). This type of functional has reduced self-interaction and the correct asymptote to the Coulomb potential, which has been shown to give improved SFI rates. ${ }^{20,43}$ The TDDFT simulation parameters are shown in Table III. For all the molecules studied, we find very good agreement between our DFT ionization potentials and experiment, ${ }^{48}$ as shown in the 5 th and 6th rows of Table IV.

In our simulations, the laser-molecule interaction is treated in the dipole approximation and length gauge. For consistency with experiments, unless otherwise specified, all simulations use a 800nm-wavelength pulse with a sine-squared electric field envelope and an intensity envelope with a $37-f$ f FWHM duration. Because each halomethane has a different ionization potential, the peak intensity is adjusted for each compound to be close to saturation, defined

TABLE IV. Computed (TD)DFT properties for $\mathrm{CH}_{3} \mathrm{X}$ : Rows: (2-4) the angle between the $\mathrm{CX}$ and $\mathrm{CH}$ bonds, and the length of the $\mathrm{CH}$ and $\mathrm{CX}$ bonds, respectively, as self-consistently computed from our DFT simulations; $(5,6)$ experimental (from NIST website) and numerical (from our DFT computations) ionization potentials, respectively; (7) numerical saturation intensities; (8) unaligned average ionization yield for that intensity and with the laser parameters listed in the text; (9) estimated total ionization leakage; (10) cycle-average hole localization on the halogen group.

\begin{tabular}{lccc}
\hline \hline & $\mathrm{CH}_{3} \mathrm{~F}$ & $\mathrm{CH}_{3} \mathrm{Cl}$ & $\mathrm{CH}_{3} \mathrm{Br}$ \\
\hline $\mathrm{H} \hat{\mathrm{CX}}(\mathrm{deg})$ & 109.0 & 108.5 & 107.9 \\
$|\mathrm{CH}|(\AA)$ & 1.091 & 1.086 & 1.085 \\
$|\mathrm{CX}|(\AA)$ & 1.378 & 1.779 & 1.934 \\
Ip exp. $(\mathrm{eV})$ & 12.50 & 11.26 & 10.54 \\
Ip DFT (eV) & 13.33 & 11.35 & 10.47 \\
$\mathrm{I}_{0}\left(\mathrm{TW} / \mathrm{cm}^{2}\right)$ & 130 & 80 & 50 \\
Yield (elec) (\%) & 14.5 & 16.7 & 12.0 \\
Leakage (elec) (\%) & 1.76 & 2.18 & 2.12 \\
Cycle avg. hole loc. (\%) & $\approx 50$ & $\approx 70$ & $\approx 75$ \\
\hline \hline
\end{tabular}




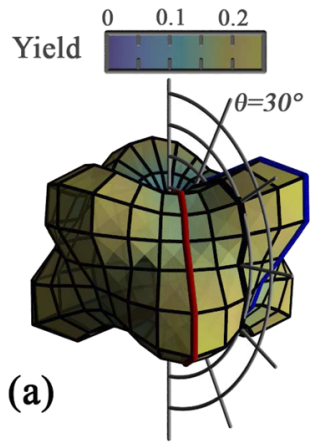

Highest-occupied shell

(b)

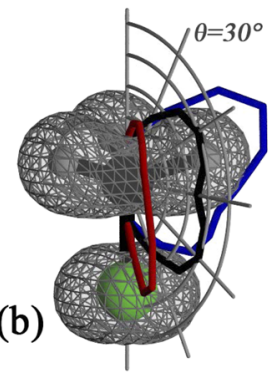

FIG. 4. (a) Full angle-resolved $\mathrm{CH}_{3} \mathrm{~F}^{+}$yield computed with TDDFT for a 130 $\mathrm{TW} / \mathrm{cm}^{2}$-intensity, 800-nm-wavelength, and a linearly polarized laser field with $37-f s$ FWHM in intensity. (b) Electronic structure in the highest-occupied shell of $\mathrm{CH}_{3} \mathrm{~F}$, defined as the combined density from the degenerate highest-occupied molecular orbitals. The isosurface threshold is set to $10^{-2}$ elec./a.u. ${ }^{3}$. For comparison, we repeat some of the ionization yield lineouts of panel (a), with the matching (color) shade, in panel (b), illustrating that the yield follows the shape of the methyl group density.

as $15 \%$ unaligned ionization yield. ${ }^{20}$ Saturation intensities and corresponding unaligned yields are listed in the 7th and 8th rows of Table IV. All ionization yields and rates (except MO populations) are corrected for a self-consistent estimate of spurious ionization leakage due to the overlap between occupied molecular orbitals and the CAPs. ${ }^{20}$ These leakage yields are listed in the 9th row of the table. At the intensities studied in this work, the "signal-to-noise" ratio of ionization to leakage is sufficiently high to carry out the analysis detailed below.

For each individual simulation, the laser polarization is chosen to be along the direction defined by spherical angles $\theta$ and $\phi$. The molecular axis, defined by the $\mathrm{C}-\mathrm{X}$ bond, is along $\theta=0$ and an $\mathrm{H}-\mathrm{C}-\mathrm{X}$ plane is located at $\phi=0$. An illustration of such a $(\theta, \phi)$ resolved yield is shown in Fig. 4(a) for $\mathrm{CH}_{3} \mathrm{~F}$, and it shows a distinct shape due to the methyl group. Experiments, on the other hand, do not control the azimuthal angle $\phi$. Thus, for consistency, and unless otherwise specified, we report data averaged over the angle $\phi$ and refer to $\theta$ as the alignment angle since it is the angle between the polarization and the molecular axis. Empirically, we have found that the ionization signal at $\phi=30^{\circ}$ gives a very good approximation of the azimuthal average. This "magic" angle, highlighted with the middle (black) lineout in Fig. 4, is a result of the symmetry of the target-plus-laser system. We have checked the robustness of this approximation for all the molecules studied here.

\section{SIMULATION RESULTS}

In Fig. 5, we compare the alignment-angle-resolved total ionization yields for three halogenated methane molecules (thick black curves). We convert our yields calculated using oriented molecules to those appropriate for aligned molecules by averaging yields for $\theta$ with $\pi-\theta$. Qualitatively, the $\mathrm{CH}_{3} \mathrm{~F}^{+}$yield exhibits an elongated bow-tie shape with a pronounced minimum near parallel alignment $\left(\theta=0^{\circ}\right)$ and a substantially larger magnitude near perpendicular alignment $\left(90^{\circ}\right)$. The $\mathrm{CH}_{3} \mathrm{Cl}^{+}$yield is markedly different, with a

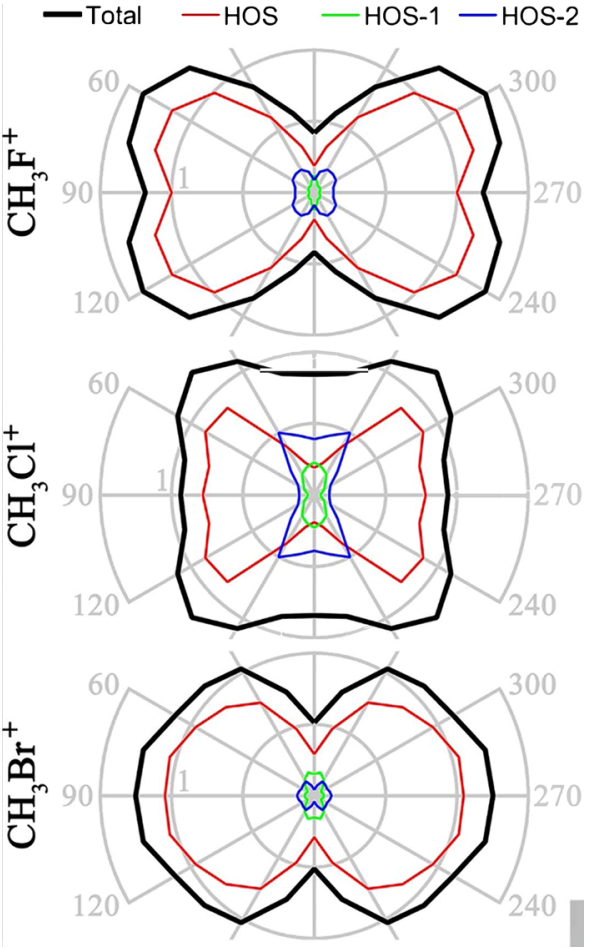

FIG. 5. Alignment-angle-resolved ionization yields for the halomethane series calculated at their respective saturation intensities (see Table IV). The orbital-resolved yields are shown in terms of the highest occupied shells (HOSs) built from the sum of degenerate orbitals. Each curve is normalized by the unaligned total yield in the corresponding compound. The observed trend of bow tie $\rightarrow$ cloverleaf $\rightarrow$ peanut shapes can be understood as going from single $\rightarrow$ double $\rightarrow$ single channel ionization.

cloverleaf shape having a shallow minimum for both parallel and perpendicular alignments. The $\mathrm{CH}_{3} \mathrm{Br}^{+}$yield is similar to $\mathrm{CH}_{3} \mathrm{~F}^{+}$, but has more of a peanut shape, with a minimum at $\theta=0^{\circ}$ and a maximum at $90^{\circ}$. These results compare well with the experimental measurements for $\mathrm{CH}_{3} \mathrm{Cl}^{+}$and $\mathrm{CH}_{3} \mathrm{Br}^{+}$discussed in Sec. III (see Fig. 3). The well-defined differences in angular yields among the halomethanes are somewhat surprising, given that all three have similar geometries and molecular orbital shapes. To elucidate the physical origins of these differences, in Secs. VI A-VI C, we interpret the TDDFT ionization results using three complementary pictures: molecular orbitals, subcycle dynamics, and hole localization.

\section{A. Molecular orbital resolved yields}

Traditionally, strong-field molecular ionization is interpreted using the shape of the frontier occupied molecular orbitals, ${ }^{1}$ typically the highest occupied molecular orbital. In this spirit, and to compare to experiments that align the molecules with respect to $\theta$ but not $\phi$, we combine the two degenerate HOMO orbitals ( $x$ and $y$ oriented) to build a highest occupied shell (HOS). For each compound, the HOS and HOS- 2 are $\pi$ orbitals and the HOS- 1 is a $\sigma$ orbital. As an example, Fig. 4(b) shows the density of the $\mathrm{HOS}$ for $\mathrm{CH}_{3} \mathrm{~F}$. It exhibits delocalized pockets of density on both ends of the molecule, split 
approximately evenly between the fluorine (43\%) and the methyl (57\%) centers. Plots of the HOS for other members of the family (not shown) are similar, with $84 \%$ and $89 \%$ of the HOS density on the halogen for $\mathrm{CH}_{3} \mathrm{Cl}$ and $\mathrm{CH}_{3} \mathrm{Br}$, respectively. For $\mathrm{CH}_{3} \mathrm{Br}$, the density on the methyl end of the molecule is concentrated around the individual hydrogen centers, while the localization of the methyl density for $\mathrm{CH}_{3} \mathrm{Cl}$ is intermediate between the fluorine and bromine cases.

Next, we use the lower lying molecular orbitals to construct lower lying energy shells, HOS-1 and HOS-2. We then compute the contribution from each of these shells to the total ionization in the three molecules, and these shell-resolved yields are also shown in Fig. 5. Since these yields are a measure of the populations after the end of the pulse, they do not give direct information about what happens during the ionization process itself, which may involve orbital polarization and Stark shifting due to the strong field. Thus, these are best viewed as qualitative measures of the multichannel nature of ionization. It is worth noting that while the calculated ionization yields are smooth with respect to angle, some of the MO contributions appear somewhat sharp (e.g., the HOS-2 contribution from $\mathrm{CH}_{3} \mathrm{Cl}$ in Fig. 5). This is likely due to a combination of the finite basis set and CAP positioning, as well as nonphysical leakage from the basis set. For $\mathrm{CH}_{3} \mathrm{~F}^{+}$and $\mathrm{CH}_{3} \mathrm{Br}^{+}$, we find that, irrespective of the alignment angle, the yield is dominated by the HOS. Deeper channels play only a minor role in these two molecules. Ionization of $\mathrm{CH}_{3} \mathrm{Cl}$, in contrast, is multichannel with significant contributions from both the HOS and HOS-2 (both $\pi$ orbitals), with nontrivial angular dependence. While the yield is dominated by the HOS near $\theta=90^{\circ}$, there are comparable contributions from HOS and HOS-2 for angles between $\theta=0$ and $45^{\circ}$, making $\mathrm{CH}_{3} \mathrm{Cl}$ a promising candidate for studies of single vs multichannel ionization. Overall, in a MO picture, we find the progression from bow tie to cloverleaf to peanut shape in the ionization yields shown in Fig. 5 can be understood as going from single channel to multichannel and then back to single channel ionization.

Contrary to what conventional Molecular Orbital-AmmosovDelone-Krainov (MO-ADK) theory ${ }^{1}$ would predict, the angleresolved shape of the ionization yields cannot be consistently explained by the HOS shape for all of the halomethanes. In particular, detailed analysis of the ionization yields shows that these differences can be traced to the differential involvement of the methyl and halogen groups. The $\mathrm{CH}_{3} \mathrm{~F}^{+}$yield, shown in Fig. 4(a), exhibits a large modulation as the $\phi$ angle is varied (approximately $40 \%$ at $\theta=60^{\circ}$ ). Comparing with the HOS density in Fig. $4(\mathrm{~b})$, we find that the yield closely matches the shape of the methyl group and its threefold symmetry, i.e., the absolute maximum of the yield is obtained when the laser polarization is nearly parallel to a $\mathrm{C}-\mathrm{H}$ bond. In addition, the $\mathrm{CH}_{3} \mathrm{~F}^{+}$yield averaged over $\phi$, shown in Fig. 5(a), exhibits a shallow (interference) minimum around $\theta=90^{\circ}$, indicating that electrons localized around both centers participate in the ionization at perpendicular alignment. We also note that the $\mathrm{CH}_{3} \mathrm{~F}^{+}$yield can be understood chemically in terms of the large electronegativity of fluorine, which makes it less likely to share electrons, leaving a larger role for the methyl group in determining the ionization yield for this molecule.

Turning to the other case where ionization is single-channel, we note again that the $\mathrm{HOS}$ electron density in $\mathrm{CH}_{3} \mathrm{Br}$ is highly (89\%) localized around bromine. As expected from this, the $\mathrm{CH}_{3} \mathrm{Br}^{+}$yield in Fig. 5(c) is consistent with ionization being mostly dictated by the halogen group. This is further confirmed by azimuthal-angleresolved yields (not shown), which have only a weak modulation $\left(\approx 6 \%\right.$ at $\left.\theta=60^{\circ}\right)$ of the yield with rotation through the angle $\phi$ around the molecular axis.

\section{B. Subcycle dynamics}

To further interpret the ionization in terms of two-centers $\left(\mathrm{CH}_{3}\right.$ and $\left.\mathrm{X}\right)$ in the halomethanes, the subcycle dynamics are computed from the normalized time-dependent ionization rates,

$$
\Gamma(\theta ; t)=\frac{\partial_{t} Y(\theta ; t)}{Y(\theta ;+\infty)},
$$

where $\partial_{t} Y(\theta ; t)$ is the instantaneous time derivative of the yield during the pulse and $Y(\theta ;+\infty)$ is the total yield after the pulse. $\Gamma(\theta ; t)$ allows us to quantify how the ionization relates to the instantaneous electric field direction. The normalization in Eq. (1) enables comparison of subcycle ionization dynamics between alignment angles that have different total yields. Because the CAP is located at some distance from the center of the molecule, there is a nontrivial delay between ionization and the loss of yield to the CAP, and the fine details of the rates are hard to interpret. Instead, we focus on general trends and their correlation with the laser-induced force at the half-laser-cycle time scale.

Ionization rates calculated over two laser cycles at the peak of the $37 \mathrm{fs}$ FWHM pulse are shown in Fig. 6 for a range of alignment angles. Results for $90^{\circ}$ are not shown because, irrespective of the target, the instantaneous rates exhibit peaks each half laser cycle, as one would expect from the absence of head-vs-tail distinction for perpendicular alignment. In the $\mathrm{CH}_{3} \mathrm{~F}^{+}$case, Fig. 6(a), there are large bursts of ionization when the component of the electric field parallel to the axis points in the positive direction, i.e., when the force on the electrons points from the halogen to the methyl. This is true for all angles between $15^{\circ}$ and $75^{\circ}$. In contrast, there is little ionization when the field points in the opposite direction. The subcycle analysis of $\mathrm{CH}_{3} \mathrm{Br}$ in Fig. 6(c) shows a similar but opposite effect, where the ionization rate is the greatest when the force on the electrons points from methyl to bromine.

In contrast, $\mathrm{CH}_{3} \mathrm{Cl}$ [Fig. 6(b)] shows more complex ionization dynamics than the other halomethanes. At angles close to perpendicular alignment, the half-cycle bursts of ionization are the largest when the force points from methyl toward chlorine [see the $75^{\circ}$ curve in Fig. 6(b)]. Conversely, for angles close to parallel alignment, the ionization rate is greater when the force points in the opposite direction. At an alignment angle of $45^{\circ}$, approximately the same amount of ionization is produced in each half cycle, regardless of the field direction. All of these results are consistent with the observation that multiple channels contribute to angle-dependent strong-field ionization of $\mathrm{CH}_{3} \mathrm{Cl}$, with mostly the HOS for perpendicular alignment, HOS-2 dominating near parallel alignment, and roughly equal amounts of HOS and HOS- 2 contributing near $45^{\circ}$. These results are also consistent with previously reported simulations of ionization of $\mathrm{CH}_{3} \mathrm{X}$ in static fields, where a similar progression from methyl to halogen-dominated angle-dependent ionization was observed.

\section{Hole localization}

Finally, we use a density-based hole localization analysis to track where, on average, the electron hole resides following 

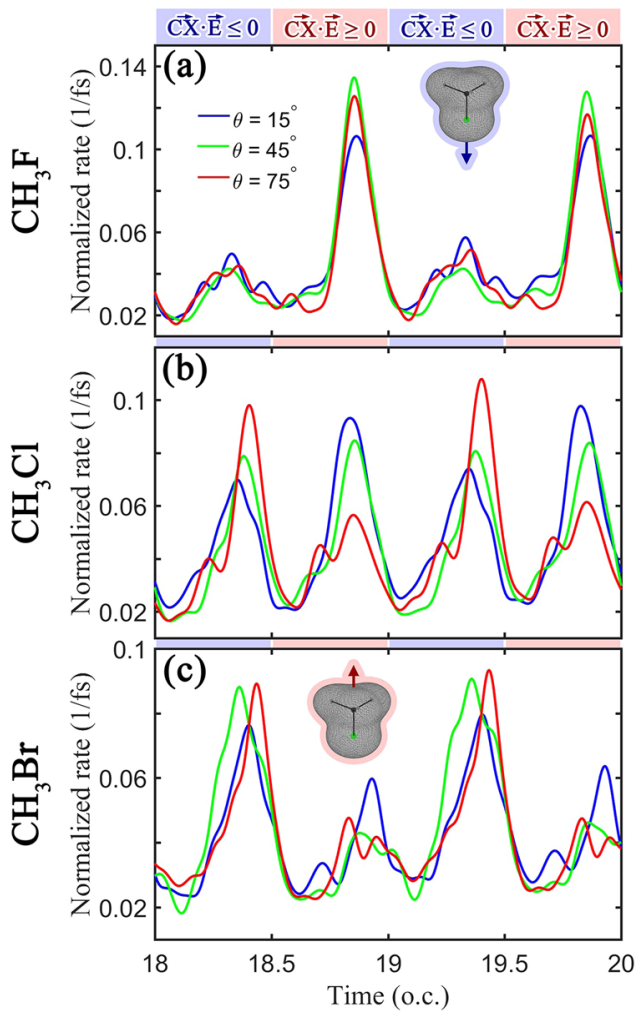

FIG. 6. Normalized instantaneous ionization rates around the peak of the laser pulse envelope for various alignment angles (see legend) for (a) $\mathrm{CH}_{3} \mathrm{~F}$, (b) $\mathrm{CH}_{3} \mathrm{Cl}$, and (c) $\mathrm{CH}_{3} \mathrm{Br}$. Each curve has been smoothed by convolution with a cosine-fourth function with 180 as FWHM duration. For each half-laser cycle, we indicate the direction of the component of the field parallel to the molecular axis with respect to the $\overrightarrow{C X}$ direction. As illustrated in the molecular insets, for $\overrightarrow{C X} \cdot \vec{E} \leq 0$, the force on the electrons due to that field component points from the methyl groups toward the halogens. For $\overrightarrow{C X} \cdot \vec{E} \geq 0$, the force component points from the halogens to the methyl groups.

ionization. This is useful both as an interpretive tool and for predicting the degree of hole localization which is likely important for triggering attosecond charge migration. This analysis involves extracting the cation dynamics from the total density by subtracting the field-induced polarization of the neutral. ${ }^{20}$ Briefly, we calculate $n_{\mathrm{CH}_{3} / \mathrm{X}}(t)$ as the time-dependent number of electrons around the methyl and halogen groups, respectively. ${ }^{49}$ For these calculations, we use a pulse that ramps up over six optical cycles and then maintains a constant intensity thereafter. During the ramp up, before the field is strong enough to induce ionization, we identify the magnitude of the transient polarization effects in the neutral component. We find that, irrespective of the alignment angle, the polarization of the neutral charge density is driven by the component of the field parallel to the molecular axis $E_{\|}$. We use this to define generalized atomic susceptibilities $\chi_{\|}$, which are the linear proportionalities between the applied field and the net charges on the halogen and methyl groups, in the absence of ionization. ${ }^{20}$ Although in principle higher order terms might be needed, these were observed to be insignificant. At a full intensity, when the laser induces ionization, we define the hole density in the cation around one or the other end of the molecule as $n_{\mathrm{CH}_{3} / \mathrm{X}}^{c}$. It is obtained by subtracting the (extrapolated) neutral-only response from the TDDFT density,

$$
\begin{aligned}
n_{\mathrm{CH}_{3}}^{c}(t) & =\frac{\Delta n_{\mathrm{CH}_{3}}(t)-\left[1-\Delta n_{t o t}(t)\right] \chi_{\|} E_{\|}}{\Delta n_{\text {tot }}(t)}, \\
n_{\mathrm{X}}^{c}(t) & =\frac{\Delta n_{\mathrm{X}}(t)+\left[1-\Delta n_{\text {tot }}(t)\right] \chi_{\|} E_{\|}}{\Delta n_{\text {tot }}(t)}
\end{aligned}
$$

where $\Delta n_{\mathrm{CH}_{3} / \mathrm{X}}=n_{\mathrm{CH}_{3} / \mathrm{X}}(-\infty)-n_{\mathrm{CH}_{3} / \mathrm{X}}(t)$ is the number of electrons "lost" around each molecular group and $\Delta n_{\text {tot }}=\Delta n_{\mathrm{CH}_{3}}+\Delta n_{\mathrm{X}}$. We have improved on the analysis in Ref. 20 by accounting for ionization-induced depletion of the neutral component with the factor $\left[1-\Delta n_{t o t}\right]$. The factor $1 / \Delta n_{t o t}$ is for normalization and charge conservation in the cation such that $n_{\mathrm{CH}_{3}}^{c}+n_{\mathrm{X}}^{c}=1$ electron hole at all times.

In Fig. 7, we compare the hole density around the methyl and halogen groups, as defined by Eq. (2), for the different members of the $\mathrm{CH}_{3} \mathrm{X}$ family. In this approach, a negative hole number corresponds to an excess of electrons compared to the field-free neutral reference. These dynamics involve both polarization of the cation (low-frequency oscillations) as well as field-driven hole dynamics within the molecule, visible as cycle-averaged hole localization and higher frequency oscillations. Thus, the meaningful quantity for these results is the cycle-averaged hole density on the fragments, which reflects the location of the hole upon ionization. Unlike the neutral contribution, where we could define an angle-independent $\chi_{\|}$, the magnitude of the oscillations in the cation depends on the alignment angle and reflects a different "mobility" for the density once a hole has been created. A detailed analysis of the cation

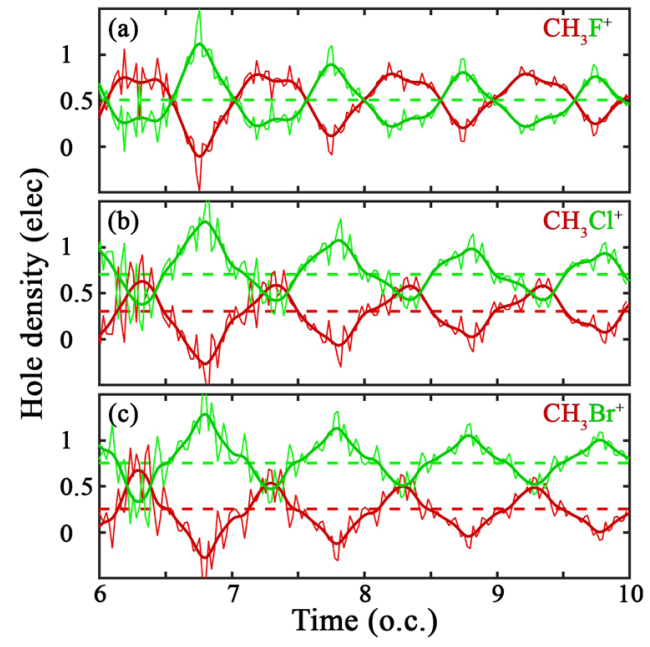

FIG. 7. Reconstructed subcycle hole density [see Eq. (2)] around the methyl (red) and halogen (green) groups for cations with (a) fluorine, (b) chlorine, and (c) bromine functionalization. In all panels, thin curves correspond to raw results from the TDDFT computation and analysis. Corresponding thick curves are obtained by convolution with a Gaussian with 1/20th laser cycle standard deviation. Horizontal dashed lines label the cycle average localization listed in the last row of Table IV. In all panels, we use a laser with a constant-intensity envelope, six-laser-cycle ramp-up, and alignment angle $\theta=45^{\circ}$ 
dynamics is, however, beyond the scope of this paper and will be the subject of future studies.

Instead, we focus on the cycle-averaged hole density on the fragments, which is a direct measurement of where the hole ends up following ionization. For $\mathrm{CH}_{3} \mathrm{~F}^{+}$, in Fig. 7 (a), we see that the average hole density is an approximately $50 \%-50 \%$ distribution between the methyl and halogen groups. This is somewhat contrary to the subcycle ionization rate analysis, which showed a strong subcycle asymmetry between half cycles. This result is possibly an indication that the hole, once created, is redistributed during the subsequent interaction with the laser. In $\mathrm{CH}_{3} \mathrm{Cl}^{+}$[Fig. 7(b)], the hole density is $\sim 70 \%$ localized on the halogen end, and in $\mathrm{CH}_{3} \mathrm{Br}^{+}$[Fig. 7(c)], it is even more so ( $75 \%$ on the bromine end). In contrast to $\mathrm{CH}_{3} \mathrm{~F}$, the $\mathrm{CH}_{3} \mathrm{Br}$ result is consistent with the $\mathrm{MO}$ and subcycle pictures. The cycle average hole densities are tabulated in the last row of Table IV.

Given that the HOS density on the halogen increases from $43 \%$ to $84 \%$ to $89 \%$ as we go from $\mathrm{F}$ to $\mathrm{Cl}$ to $\mathrm{Br}$, it is perhaps not surprising that the hole localization increases from $50 \%$ to $70 \%$ to $75 \%$ with the same functionalization change. Thus, the intuitive result from this analysis is that SFI in halogenated molecules will likely result in better hole localization as one goes to heavier elements $\mathrm{F} \rightarrow \mathrm{Cl} \rightarrow \mathrm{Br} \rightarrow \mathrm{I}$.

\section{SUMMARY AND CONCLUSIONS}

In summary, we have measured and simulated strong-field single ionization in chloro- and bromomethane as a function of laser polarization angle relative to the $\mathrm{C}-\mathrm{X}$ axis. In addition, we have carried out TDDFT simulations for flouromethane for a more complete view of the halomethanes. The saturation intensities and angle-dependent yields agree well between TDDFT calculations and experiments, with both experiment and theory showing a cloverleaf shaped yield for $\mathrm{CH}_{3} \mathrm{Cl}^{+}$and a peanut shape for $\mathrm{CH}_{3} \mathrm{Br}^{+}$. The halomethane family offers an illustrative example of the effects of chemical functionalization on SFI in molecules. Despite having identical symmetries and similar frontier orbital shapes, there are substantial differences in the mechanisms of ionization. Through our simulations, this key result can be viewed in three complementary ways.

First, projection of the surviving electron density onto molecular orbitals shows that $\mathrm{SFI}$ in $\mathrm{CH}_{3} \mathrm{Br}$ and $\mathrm{CH}_{3} \mathrm{~F}$ is largely single-channel, dominated by the HOMO. Conversely, SFI of $\mathrm{CH}_{3} \mathrm{Cl}$ involves multiple channels, and the predominant contributor depends upon the alignment angle. This manifests as a bow tie shaped distribution that cannot be predicted simply from the shape of the HOMO. We conclude that molecular SFI calculations and interpretations based solely on the shape of a single orbital (e.g., the HOMO) can be problematic.

Second, analysis of the subcycle dynamics indicates that in $\mathrm{CH}_{3} \mathrm{~F}$, the SFI rate is the greatest when the applied force on the electrons points from the fluorine toward the methyl group. Conversely, $\mathrm{CH}_{3} \mathrm{Br}$ is more readily ionized when this force points from the methyl group toward the $\mathrm{Br}$ atom. In $\mathrm{CH}_{3} \mathrm{Cl}$, the dependence of the ionization rate on the laser force direction actually reverses as the alignment angle varies from near $0^{\circ}$ to near $90^{\circ}$. Future experiments combining transiently oriented molecules with an asymmetric twocolor ionizing field might test the TDDFT predictions regarding the striking differences in the preferred field direction for maximizing SFI rates in these three molecules.

Third, our simulations show that with increasing halogen mass, the hole produced during SFI of halomethanes is progressively more localized on the halogen atom. This has potential implications for SFI-triggered attosecond dynamics, for example, where creating a localized hole is likely critical to initiating observable charge migration.

Taken together, the theoretical results of the MO and subcycle analyses offer a consistent picture, allowing us to infer other key aspects of the SFI dynamics in halomethanes that cannot be directly obtained from the calculations or experiments. In particular, in all cases, ionization appears to favor direct tunneling of electrons out of the molecule, rather than some intramolecular tunneling process. This conclusion is supported by the following observations. The simulations indicate that ionization of $\mathrm{CH}_{3} \mathrm{~F}$ is dominated by electrons that are initially localized on the methyl group (given the strong $\phi$-dependence), and the ionization rate is the greatest when the force on the electrons points from $\mathrm{CH}_{3}$, out of the molecule. In $\mathrm{CH}_{3} \mathrm{Br}$, ionization is dominated by electrons in the HOS that are initially localized on $\mathrm{Br}$ and proceeds more rapidly when the force on the electrons points from $\mathrm{Br}$, out of the molecule. In the intermediate case of $\mathrm{CH}_{3} \mathrm{Cl}$, the ionization evolves from $\mathrm{CH}_{3} \mathrm{~F}$-like for $\theta=0$ to $\mathrm{CH}_{3} \mathrm{Br}$-like for angles closer to $\theta=90^{\circ}$, with no substantial directional preference for $\theta \simeq 45^{\circ}$. Thus, while the preferred direction of ionization (relative to the permanent molecular dipole) appears to reverse with the alignment angle, the tunneling is always direct. The angular dependence derives from the fact that the HOS density (which dominates the ionization near perpendicular alignment) is localized on $\mathrm{Cl}$, while the HOS-2 density (which is the primary contributor to the ionization yield for parallel alignment) is more localized on the methyl group. Moving forward, this mechanistic picture in terms of orbital contributions and orientationdependent yields should be invaluable for interpreting and guiding two-color experiments aimed at exploring directional ionization and control of charge-migration dynamics in halogen-functionalized molecules.

\section{ACKNOWLEDGMENTS}

This work was supported by the U.S. Department of Energy, Office of Science, Basic Energy Sciences, under Award No. DESC0012462. Portions of this research were conducted with high performance computational resources provided by Louisiana State University (www.hpc.lsu.edu) and the Louisiana Optical Network Infrastructure (www.loni.org).

\section{REFERENCES}

${ }^{1}$ X. M. Tong, Z. X. Zhao, and C. D. Lin, "Theory of molecular tunneling ionization," Phys. Rev. A 66, 033402 (2002).

${ }^{2}$ I. V. Litvinyuk, K. F. Lee, P. W. Dooley, D. M. Rayner, D. M. Villeneuve, and P. B. Corkum, “Alignment-dependent strong field ionization of molecules," Phys. Rev. Lett. 90, 233003 (2003).

${ }^{3}$ D. Pinkham and R. R. Jones, "Intense laser ionization of transiently aligned CO," Phys. Rev. A 72, 023418 (2005).

${ }^{4}$ D. Pavičić, K. F. Lee, D. M. Rayner, P. B. Corkum, and D. M. Villeneuve, "Direct measurement of the angular dependence of ionization for $\mathrm{N}_{2}, \mathrm{O}_{2}$, and $\mathrm{CO}_{2}$ in intense laser fields," Phys. Rev. Lett. 98, 243001 (2007). 
${ }^{5}$ E. P. Fowe and A. D. Bandrauk, "Nonperturbative time-dependent densityfunctional theory of ionization and harmonic generation in OCS and $\mathrm{CS}_{2}$ molecules with ultrashort intense laser pulses: Intensity and orientational effects," Phys. Rev. A 84, 035402 (2011).

${ }^{6}$ J. L. Hansen, L. Holmegaard, J. H. Nielsen, H. Stapelfeldt, D. Dimitrovski, and L. B. Madsen, "Orientation-dependent ionization yields from strong-field ionization of fixed-in-space linear and asymmetric top molecules," J. Phys. B: At., Mol. Opt. Phys. 45, 015101 (2012).

${ }^{7}$ E. P. Fowe and A. D. Bandrauk, "Nonperturbative time-dependent density functional theory (TDDFT) and time-dependent electron localization function (TDELF) study of the ionization of OCS and $\mathrm{CS}_{2}$ with ultrashort intense laser pulses," Can. J. Chem. 90, 616 (2012).

${ }^{8}$ L. B. Madsen, F. Jensen, O. I. Tolstikhin, and T. Morishita, "Structure factors for tunneling ionization rates of molecules," Phys. Rev. A 87, 013406 (2013).

${ }^{9}$ A. Russakoff, S. Bubin, X. Xie, S. Erattupuzha, M. Kitzler, and K. Varga, "Time-dependent density-functional study of the alignment-dependent ionization of acetylene and ethylene by strong laser pulses," Phys. Rev. A 91, 023422 (2015).

${ }^{10}$ S. G. Walt, N. B. Ram, A. v. Conta, O. I. Tolstikhin, L. B. Madsen, F. Jensen, and H. J. Wörner, "Role of multi-electron effects in the asymmetry of strong-field ionization and fragmentation of polar molecules: The methyl halide series," J. Phys. Chem. A 119, 11772-11782 (2015)

${ }^{11}$ R. Johansen, K. G. Bay, L. Christensen, J. Thøgersen, D. Dimitrovski, L. B. Madsen, and H. Stapelfeldt, "Alignment-dependent strong-field ionization yields of carbonyl sulfide molecules induced by mid-infrared laser pulses," J. Phys. B: At., Mol. Opt. Phys. 49, 205601 (2016).

${ }^{12}$ V. Makhija, X. Ren, D. Gockel, A.-T. Le, and V. Kumarappan, “Orientation resolution through rotational coherence spectroscopy,” e-print arXiv:1611.06476v2 (2016).

${ }^{13}$ K. J. Schafer, B. Yang, L. F. DiMauro, and K. C. Kulander, “Above threshold ionization beyond the high harmonic cutoff," Phys. Rev. Lett. 70, 1599 (1993).

${ }^{14} \mathrm{P}$. B. Corkum, "Plasma perspective on strong field multiphoton ionization," Phys. Rev. Lett. 71, 1994 (1993).

${ }^{15}$ P. B. Corkum and F. Krausz, “Attosecond science," Nat. Phys. 3, 381 (2007).

${ }^{16}$ F. Krausz and M. Ivanov, "Attosecond physics," Rev. Mod. Phys. 81, 163 (2009).

${ }^{17}$ M. Meckel, D. Comtois, D. Zeidler, A. Staudte, D. Pavičić, D. H. C. Bandulet, H. Pépin, J. C. Kieffer, R. Dörner, D. M. Villeneuve, and P. B. Corkum, "Laserinduced electron tunneling and diffraction," Science 320, 1478 (2008).

${ }^{18}$ C. I. Blaga, J. Xu, A. D. DiChiara, E. Sistrunk, K. Zhang, P. Agostini, T. A. Miller, L. F. DiMauro, and C. D. Lin, "Imaging ultrafast molecular dynamics with laserinduced electron diffraction," Nature 483, 194 (2012).

${ }^{19}$ J. Xu, C. I. Blaga, K. Zhang, Y. H. Lai, C. D. Lin, T. A. Miller, P. Agostini, and L. F. DiMauro, "Diffraction using laser-driven broadband electron wave packets," Nat. Commun. 5, 4635 (2014).

${ }^{20}$ P. Sándor, A. Sissay, F. Mauger, P. M. Abanador, T. T. Gorman, T. D. Scarborough, M. B. Gaarde, K. Lopata, K. J. Schafer, and R. R. Jones, “Angle dependence of strong-field single and double ionization of carbonyl sulfide," Phys. Rev. A 98, 043425 (2018)

${ }^{21} \mathrm{P}$. Hoerner and H. B. Schlegel, "Angular dependence of strong field ionization of $\mathrm{CH}_{3} \mathrm{X}(\mathrm{X}=\mathrm{F}, \mathrm{Cl}, \mathrm{Br}$, or I) using time-dependent configuration interaction with an absorbing potential,” J. Phys. Chem. A 121, 5940-5946 (2017).

${ }^{22}$ S. M. Hankin, D. M. Villeneuve, P. B. Corkum, and D. M. Rayner, "Intense-field laser ionization rates in atoms and molecules," Phys. Rev. A 64, 013405 (2001).

${ }^{23}$ S. Luo, W. Hu, J. Yu, X. Li, L. He, C. Wang, F. Liu, and D. Ding, "Multielectron effects in the strong field sequential ionization of aligned $\mathrm{CH}_{3} \mathrm{I}$ molecules," J. Phys. Chem. A 121, 6547-6553 (2017).

${ }^{24}$ A. H. Winney, G. Basnayake, D. A. Debrah, Y. F. Lin, S. K. Lee, P. Hoerner, Q. Liao, H. B. Schlegel, and W. Li, "Disentangling strong-field multielectron dynamics with angular streaking," J. Phys. Chem. Lett. 9, 2539-2545 (2018).

${ }^{25}$ C. Marceau, V. Makhija, D. Platzer, A. Y. Naumov, P. B. Corkum, A. Stowlow, D. M. Villeneuve, and P. Hockett, "Molecular frame reconstruction using timedomain photoionization interferometry," Phys. Rev. Lett. 119, 083401 (2017).

${ }^{26}$ R. Trebino, K. W. DeLong, D. N. Fittinghoff, J. N. Sweetser, M. A. Krumbügel, B. A. Richman, and D. J. Kane, "Measuring ultrashort laser pulses in the time-frequency domain using frequency-resolved optical gating," Rev. Sci Instrum. 68, 3277-3295 (1997).

${ }^{27}$ U. Even, J. Jortner, D. Noy, N. Lavie, and C. Cossart-Magos, "Cooling of large molecules below $1 \mathrm{~K}$ and He clusters formation," J. Chem. Phys. 112, 8068-8071 (2000).

${ }^{28}$ K. Egodapitiya, S. Li, and R. R. Jones, "Terahertz-induced field-free orientation of rotationally excited molecules,” Phys. Rev. Lett. 112, 103002 (2014).

${ }^{29}$ M. Buyong, L. Jenn-Huei, and N. L. Allinger, "Molecular polarizabilities and induced dipole moments in molecular mechanics," J. Comput. Chem. 21, 813-825 (2000).

30 "NIST computational chemistry comparison and benchmark database," in NIST Standard Reference Database Number 101, Release 19, edited by R. D. Johnson III (National Institute of Standards and Technology, Gaithersburg, MD, 2018).

${ }^{31}$ R. Bocquet, D. Boucher, J. Demaison, G. Wlodarczak, and G. Graner, "The ground-state rotational constants of methyl bromide," Europhys. Lett. 2, 275 (1986).

${ }^{32} \mathrm{H}$. Ohmura, F. Ito, and M. Tachiya, "Phase-sensitive molecular ionization induced by a phase-controlled two-color laser field in methyl halides," Phys. Rev. A 74, 043410 (2006).

${ }^{33} \mathrm{~J}$. Theilhaber, " $A b$ initio simulations of sodium using time-dependent densityfunctional theory,” Phys. Rev. B 46, 12990-13003 (1992).

${ }^{34} \mathrm{~K}$. Yabana and G. F. Bertsch, "Time-dependent local-density approximation in real time," Phys. Rev. B 54, 4484-4487 (1996).

${ }^{35}$ X. Li, S. M. Smith, A. N. Markevitch, D. A. Romanov, R. J. Levis, and H. B. Schlegel, "A time-dependent Hartree-Fock approach for studying the electronic optical response of molecules in intense fields," Phys. Chem. Chem. Phys. 7, 233-239 (2005).

${ }^{36}$ W. Liang, C. T. Chapman, and X. Li, "Efficient first-principles electronic dynamics,”J. Chem. Phys. 134, 184102 (2011).

${ }^{37}$ A. Castro, H. Appel, M. Oliveira, C. A. Rozzi, X. Andrade, F. Lorenzen, M. A. L. Marques, E. K. U. Gross, and A. Rubio, "Octopus: A tool for the application of time-dependent density functional theory,” Phys. Status Solidi B 243, 2465-2488 (2006).

${ }^{38}$ M. R. Provorse and C. M. Isborn, "Electron dynamics with real-time timedependent density functional theory," Int. J. Quantum Chem. 116, 739-749 (2016).

${ }^{39}$ M. Valiev, E. J. Bylaska, N. Govind, K. Kowalski, T. P. Straatsma, H. J. Van Dam, D. Wang, J. Nieplocha, E. Apra, T. L. Windus et al., "NWChem: A comprehensive and scalable open-source solution for large scale molecular simulations," Comput. Phys. Commun. 181, 1477-1489 (2010).

${ }^{40}$ K. Lopata and N. Govind, "Modeling fast electron dynamics with real-time time-dependent density functional theory: Application to small molecules and chromophores," J. Chem. Theory Comput. 7, 1344 (2011).

${ }^{41}$ P. Krause, J. A. Sonk, and H. B. Schlegel, "Strong field ionization rates simulated with time-dependent configuration interaction and an absorbing potential," J. Chem. Phys. 140, 174113 (2014).

${ }^{42}$ P. Krause and H. B. Schlegel, "Angle-dependent ionization of small molecules by time-dependent configuration interaction and an absorbing potential," J. Phys. Chem. Lett. 6, 2140-2146 (2015).

${ }^{43}$ A. Sissay, P. Abanador, F. Mauger, M. B. Gaarde, K. J. Schafer, and K. Lopata, "Angle-dependent strong-field molecular ionization rates with tuned rangeseparated time-dependent density functional theory," J. Chem. Phys. 145, 094105 (2016).

${ }^{44}$ P. Hoerner and H. B. Schlegel, "Angular dependence of strong field ionization of haloacetylenes $\mathrm{HCCX}(\mathrm{X}=\mathrm{F}, \mathrm{Cl}, \mathrm{Br}, \mathrm{I})$, using time-dependent configuration interaction with an absorbing potential," J. Phys. Chem. C 122, 13751-13757 (2018).

${ }^{45}$ R. Baer and D. Neuhauser, "Density functional theory with correct long-range asymptotic behavior,” Phys. Rev. Lett. 94, 043002 (2005).

${ }^{46}$ R. Baer, E. Livshits, and U. Salzner, “Tuned range-separated hybrids in density functional theory," Annu. Rev. Phys. Chem. 61, 85 (2010).

${ }^{47}$ A. Karolewski, L. Kronik, and S. Kümmel, "Using optimally tuned range separated hybrid functionals in ground-state calculations: Consequences and caveats," J. Chem. Phys. 138, 204115 (2013). 
${ }^{48}$ S. Lias, "Ionization energy evaluation," in NIST Chemistry WebBook, NIST Standard Reference Database Number 69, edited by P. Linstrom and W. Mallard (National Institute of Standards and Technology, Gaithersburg, MD, 2018).

${ }^{49}$ Unlike in Ref. 20, we find that adaptive partitioning using the Bader method is unstable. We attribute this to the more complex, three-dimensional geometry of the molecule. Instead, we use a fixed boundary between the methyl and halogen groups, defined as a plane perpendicular to the CX bond. We have checked the robustness of our results with the position of that dividing the plane. The results shown in Fig. 4 use a plane located at a local minimum in the transverse density in the HOS. 